\title{
Histopathological Changes of the Testis in Rats Caused by Subcutaneous Injection of 2-Bromopropane
}

\author{
Minoru OMUrA ${ }^{1}$, Yesid Romero', Mangen ZhaO ${ }^{1}$ and Naohide INOUE ${ }^{2}$ \\ ${ }^{1}$ Department of Hygiene, Kyushu University and \\ ${ }^{2}$ Department of Hygiene, Faculty of Medicine, Kyushu University
}

\begin{abstract}
Histopathological Changes of the Testis in Rats Caused by Subcutaneous Injection of 2Bromopropane: Minoru OmuRa, et al. Department of Hygiene, Kyushu University-Mature male rats were injected subcutaneously with $1,355 \mathrm{mg} / \mathrm{kg}$ of 2-bromopropane five days a week for two weeks. In addition to the routine examinations concerning the effect on male reproductive system, we carried out an evaluation of change in the numbers of various types of germ cells in the seminiferous tubule at stages I, V, VII, X and XII to clarify germ cells which were affected by 2-bromopropane. 2-Bromopropane caused mild atrophy of the seminal vesicle, but did not show any adverse effects on spermatid/sperm count, sperm motility or sperm morphology. In the histopathological examination of the testis, the numbers of spermatogonia, preleptotene spermatocyte, leptotene spermatocyte, zygotene spermatocyte and pachytene spermatocyte at stages I and V decreased in the rats treated with 2-bromopropane. However, the numbers of pachytene spermatocyte at stages VII, $X$ and XII and round spermatids in these rats were comparable with those in control rats. In two weeks, spermatocytes develop to pachytene spermatocyte at stage VII or more developed germ cells in rats. Therefore, it seemed likely that 2-bromopropane had no adverse effects on spermatocytes but affected spermatogonia, assuming that 2-bromopropane affected germ cells immediately after the injection. In conclusion, we estimated that spermatogonia were the target cells of 2-bromopropane in the testis in rats.
\end{abstract}

(J Occup Health 1997: 39: 234-239)

Key words: 2-Bromopropane (Isopropyl bromide), Testicular toxicity. Spermatogonia, Spermatocyte, Quantitative histopathological examination, Subcutaneous injection, Rats

Received March 10, 1997; Accepted April 22, 1997

Correspondence to: $M$. Omura, Department of Hygiene, Kyushu University, Fukuoka 812-82, Japan

A part of this article has appeared in this journal as a rapid communication, Omura $M$, et al. Toxicity of 2-Bromopropane on Spermatogonia and Spermatocyte, J Occup Health 1997; 39: 1-2.
In 1995 in Korea, workers in an electronic company suffered from unexpected health hazards. Of the 33 workers ( 8 men and 25 women), more than two-third showed oligozoospermia or amenorrhea, and pancytopenia was also found among them ${ }^{1,2)}$ From an epidemiological survey, 2-bromopropane (2-BP) was suspected to be a causative chemical of these health disorders. At present, many researchers are trying to clarify the toxicities of $2-\mathbf{B P}^{3-8)}$. As for the male reproductive toxicity, Ichihara and colleagues $^{3.4)}$ first reported that 2-BP had toxic effects on the testis in rats. They exposed male rats to $300 \mathrm{ppm}$ or $1,000 \mathrm{ppm}$ of 2 -BP eight hours a day for nine weeks and found that 2-BP reduced weight of male reproductive organs, number of sperm and percentage of motile sperm and increased the incidences of morphologically abnormal sperm $^{3,4)}$. They also reported a severe atrophy of the seminiferous tubule and other severe histopathological changes of the testis caused by $2-\mathbf{B P}^{4)}$. As for the female reproductive toxicity, Kamijima and colleagues $^{5)}$ found 2-BP-induced disruption of ovarian cyclicity in rats, which may relate with an amenorrhea in workers exposed to 2-BP.

We carried out this study to add one more information to the knowledge concerning the toxicities of 2-BP, the type of germ cell in the testis which is affected by $2-B P$. In this study, we administered 2$B P$ to rats by a subcutaneous injection for two weeks. In addition to the routine examinations, we carried out an evaluation of changes in the numbers of various types of germ cells in the seminiferous tubule to clarify germ cells which were affected by 2-BP.

\section{Materials and Methods}

Animals: Eight male Wistar rats (Kud: Wistar) were purchased at 10 weeks of age and were acclimated to the animal facility for one week. Rats were housed four per cage. They were provided 
with CE-2 (Clea Japan Inc., Tokyo, Japan) and tap water ad libitum.

Treatment: All rats, 11 weeks of age, a mean body weight of $371 \mathrm{~g}(345 \mathrm{~g}-396 \mathrm{~g})$ were divided into the 2-BP group and control group of four rats each. In the 2-BP group, $1,355 \mathrm{mg} / \mathrm{kg}$ of $2-\mathrm{BP}$ (Tokyo Kasei Kogyo Co., Ltd., the purity was $>99 \%$ ) was injected subcutaneously five days a week for two weeks. Dosage of 2-BP at 1,000-ppm exposure level is calculated to be approximately $1,355 \mathrm{mg} / \mathrm{kg} /$ day, assuming that the exposure is carried out for eight hours/day and respiratory minute volume is $215 \mathrm{ml} / \mathrm{min} / 383 \mathrm{~g}$ body weight for rat $^{9)}$. Duration of one cycle of the seminiferous epithelium is about 13 days in rats $^{10)}$. Therefore, $1,355 \mathrm{mg} / \mathrm{kg}$ of $2-\mathrm{BP}$ was administered repetitively for two weeks in this study. The control rats received $1 \mathrm{ml} / \mathrm{kg}$ of saline in the same manner.

About $20 \mathrm{hr}$ after the last injection, rats were sacrificed using over dose of ether. Both sides of the testes and the epididymes, and the seminal vesicle were dissected out and were weighed. We weighed the seminal vesicle after removing secretion in this organ.

Spermatid/sperm count: The left testis was decapsulated and the left epididymis was divided into two portions (caput + corpus and cauda). Each part was homogenized in STM solution $(8.75 \mathrm{~g}$ $\mathrm{NaCl}, 0.5 \mathrm{~m} /$ Triton $\mathrm{X}-100$ and $0.1 \mathrm{~g}$ sodium ethylmercurithiosalicylate were dissolved in distilled water for 1 liter of STM solution) with a Waring blender. After that, homogenization-resistant spermatids or sperm were counted using hemocytometer.

Sperm motility and morphology: Immediately after the weighing, the right epididymis was placed in a glass petri dish containing $10 \mathrm{ml}$ of warmed $\left(37^{\circ} \mathrm{C}\right.$ ) medium. PBS (Whittaker M.A. Bioproducts, U.S.A.) containing $0.5 \%$ of BSA was used for the medium. The cauda epididymidis was opened by a razor and sperm were allowed to flow out and diffuse into the medium for three hours at $37^{\circ} \mathrm{C}$. One drop of the sperm sample was placed on a glass slide and was gently covered with a thin cover glass (Matsunami Neo Micro Cover Glass, 24 $\times 32 \mathrm{~mm}$, Thickness NO. 1, Matsunami Glass Ind., Ltd., Japan). Sperm sample was videotaped from microscope $(50 \times)$ equipped with a stage warmer (MATS-505S, Tokai Hit Co., Ltd., Japan) at $37^{\circ} \mathrm{C}$. Twelve fields were videotaped for $20 \mathrm{sec} /$ field. Analysis was performed during playback at a later time. Motile and immotile sperm were counted for all those fields and percentage of motile sperm was calculated. For the evaluation of sperm morphology, a smear of the sperm sample was made on the glass slide and stained with hematoxylin. For each smear, 1,000 sperm were examined and the sperm with morphological abnormalities were counted. Morphologically abnormal sperm were classified according to the classification of Mori et al. ${ }^{11)}$

Histopathology of the testis: The right testis was fixed in Bouin's solution, embedded in paraffin, thinly sectioned and stained with periodic acid Schiff reagent (PAS) and hematoxylin. In the histopathological examination of the testis, stages of the seminiferous tubules (stages I-XIV) were classified according to the method of Russell et al. ${ }^{12)}$ The numbers of germ cells and Sertoli cell nuclei were counted at stages I, V, VII, X and XII. A total of 10 round or ovoid seminiferous tubules were randomly examined at stages I, VII and XII and a total of five tubules were examined at stages $\mathrm{V}$ and $\mathrm{X}$. The germ cell types counted included spermatogonia, preleptotene spermatocyte, leptotene spermatocyte, zygotene spermatocyte, pachytene spermatocyte and round spermatid. The data were evaluated as the number of germ cell per one Sertoli cell nucleus per tubule. A mean value in 10 tubules (stages I, VII and XII) or five tubules (stages V and $X$ ) was used as a representative value of each animal.

Statistical analysis: Statistical difference was analyzed with Student's t-test except for the incidences of morphologically abnormal sperm. In the case of the incidences of morphologically abnormal sperm, Mann-Whitney test was used for statistical analysis. The results were interpreted as significant below a level of 0.05 .

\section{Results}

Body weight and the reproductive organ weight

Table 1 shows body weight and weights of the testes, epididymes and seminal vesicle at the sacrifice. Body weight gain was suppressed by 2-BP

Table 1. Body weight and the reproductive organ weights of the rats treated with $1,355 \mathrm{mg} / \mathrm{kg}$ of 2-bromopropane by s.c. injection five days a week for two weeks

\begin{tabular}{lcl}
\hline \multicolumn{1}{c}{ Group } & Control & 2-Bromopropane \\
\hline Body weight $(\mathrm{g})$ & $428.6 \pm 3.9$ & $375.4+17.0^{* *}$ \\
Absolute organ weight $(\mathrm{g})$ & $3.471 \pm 0.214$ & $3.077 \pm 0.280$ \\
Testes & $1.061 \pm 0.035$ & $1.018 \pm 0.061$ \\
Epididymes & $0.780 \pm 0.126$ & $0.540 \pm 0.034^{*}$ \\
Seminal vesicle & \multicolumn{3}{c}{} \\
Relative organ weight $(\mathrm{g} / 100 \mathrm{~g}$ body weight $)$ \\
Testes & $0.810 \pm 0.053$ & $0.820 \pm 0.059$ \\
Epididymes & $0.248 \pm 0.010$ & $0.271 \pm 0.008^{* *}$ \\
Seminal vesicle & $0.182 \pm 0.029$ & $0.144 \pm 0.011$ \\
\hline
\end{tabular}

Results are expressed as mean ${ }^{-}-\mathrm{SD}(\mathrm{n}=4)$. Statistical significance is analyzed with Student's $t$-Lest; ${ }^{*} p<0.05,{ }^{* *} \mathrm{p}<0.01$. 
treatment $(+16.3 \pm 5.3 \mathrm{~g}$ in the 2 -BP group vs. + $45.8 \mathrm{~g} \pm 8.7 \mathrm{~g}$ in control group) and mean body weight of the 2-BP-treated rats decreased significantly compared with that of control rats at the sacrifice (Table 1, 13\% decrease). As for the weight of the reproductive organ, relative weight of the epididymes increased significantly in the 2-BP group, though it seemed likely that this was due to a suppression of body weight gain in this group. More than $30 \%$ decrease was found in absolute weight of the seminal vesicle and a decrease in relative weight of this organ was still marginally significant $(\mathrm{p}=0.05)$ in the 2-BP group.

\section{Spermatid/sperm count, sperm motility and sperm morphology}

Table 2 shows spermatid/sperm count, \% motile sperm and the incidences of morphologically abnormal sperm. Absolute spermatid count (per testis) decreased significantly in the 2 -BP group ( $12 \%$ decrease), but relative spermatid count (per g testis) was comparable with that in control group. 2-BP did not show any adverse effects on the epididymal sperm concerning sperm count, percentage of motile sperm and sperm morphology.

\section{Histopathology of the testis}

In the 2-BP group, only a few seminiferous tubules were atrophic and severe histopathological changes such as sloughing of seminiferous epitheli-

Table 2. Spermatid/sperm count, $\%$ sperm motility and incidences of morphologically abnormal sperm in the rats treated with $1,355 \mathrm{mg} / \mathrm{kg}$ of 2 -bromopropane by s.c. injection five days a week for two weeks

\begin{tabular}{|c|c|c|}
\hline Group & Control & 2-Bromopropane \\
\hline \multicolumn{3}{|l|}{ Spermatid count ${ }^{a}$} \\
\hline$\left(\times 10^{6} /\right.$ testis $)$ & $230.3=12.4$ & $202.4+15.3^{*}$ \\
\hline$\left(\times 10^{6} / \mathrm{g}\right.$ testis $)$ & $131.4=7.3$ & $130.5 \pm 16.2$ \\
\hline \multicolumn{3}{|l|}{ Sperm count ${ }^{a}$} \\
\hline ( $\times 10^{6} /$ epididymis $)$ & $239.6 \pm 39.1$ & $265.9 \pm 26.3$ \\
\hline ( $\times 10^{6} / \mathrm{g}$ epididymis) & $466.4 \pm 67.5$ & $536.2 \pm 69.3$ \\
\hline ( $\times 10^{6} /$ cauda epididymis) & $137.6+30.8$ & $156.4 \pm 23.7$ \\
\hline$\%$ motile sperm ${ }^{2}$ & $72.9 \pm 11.8$ & $78.6 \pm 7.3$ \\
\hline \multicolumn{3}{|c|}{ Incidences of morphologically abnormal sperm $(/ 1,000 \text { sperm })^{b}$} \\
\hline Abnormal head & $7.0(1-10)$ & $3.0(1-9)$ \\
\hline Banana like & $2.5(0-4)$ & $1.5(0-7)$ \\
\hline Straight & $1.0(0-2)$ & $0.5(0-1)$ \\
\hline Teratic & $1.0\left(0^{-7}\right)$ & $1.0(0-2)$ \\
\hline Without a tail & $36.0(19-132)$ & $18.5(13-26)$ \\
\hline
\end{tabular}

${ }^{a}$ Results are expressed as mean $\pm S D(n-4)$. Statistical significance is analyzed with Student's $t$-test; ${ }^{*} \mathrm{p}<0.05$. ' Results are expressed as median (range) $(n-4)$. Statistical significance is analyzed with Mann-Whitney test. um and appearance of multinucleated giant cell were rarely observed. The number of Leydig cell in the interstitial tissue did not decrease, either. But the numbers of spermatogonia and four types of spermatocytes decreased significantly in this group. Figure 1 shows the seminiferous tubule at stage $I$ in control rat (a) and the 2-BP-treated rat (b). Normally, elongated spermatids, round spermatids and pachytene spermatocytes (arrow) are observed and spermatogonia (type $\mathrm{A}$ and intermediate type, asterisk) are situated at the base of the seminiferous epithelium at this stage (Fig. 1a). In the 2-BP-treated rats, the number of pachytene spermatocyte decreased apparently and few spermatogonia were observed at the base of the epithelium (Fig. 1b). Figure 2 shows the seminiferous epithelium of the tubule at stage VII in control rat (a) and the 2BP-treated rat (b). In control rat, elongated spermatids, round spermatids and pachytene spermatocytes were arranged regularly, and preleptotene spermatocytes (asterisk) and a few spermatogonia were situated at the base of the seminiferous epithelium (Fig. 2a). In the 2-BP-treated rats, the numbers of spermatids and pachytene spermatocyte were comparable with those in control rat. But no preleptotene spermatocyte was found at the base of the epithelium (Fig. 2b). Table 3 shows the numbers of spermatogonia, spermatocytes and round spermatid in the seminiferous tubules at stages I, V, VII, X and XII in control group, and
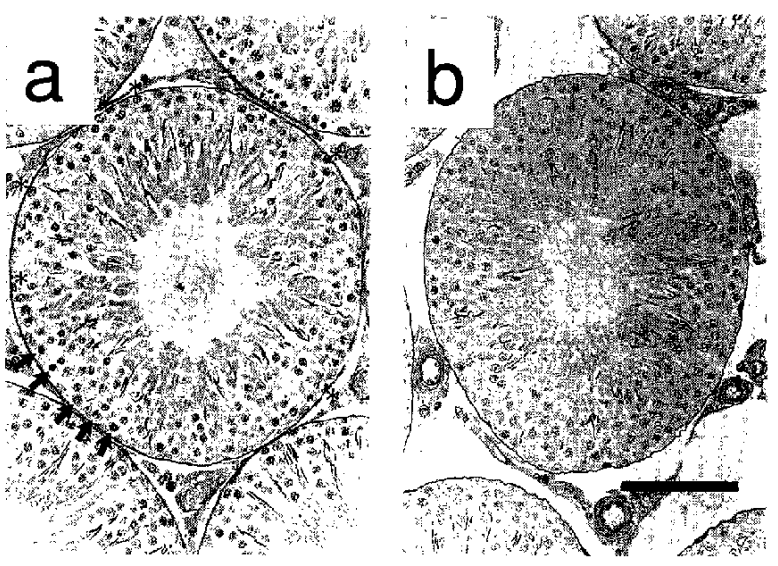

Fig. 1. Seminiferous tubule at stage $I$ in control rat (a) and the rat treated with $1,355 \mathrm{mg} / \mathrm{kg}$ of 2-bromopropane by s.c. injection five days a week for two weeks (b). The number of pachytene spermatocyte decreases in the 2 bromopropane-treated rat $(2.316 /$ Sertoli cell nucleus (a) vs. $1.080 /$ Sertoli cell nucleus (b)). And there are no spermatogonia in the tubule of 2-bromopropanetreated rat. Part of pachytene spermatocytes are indicated by arrow and spermatogonia are indicated by asterisk in control rat. Bar indicates $100 \mu \mathrm{m}$. 


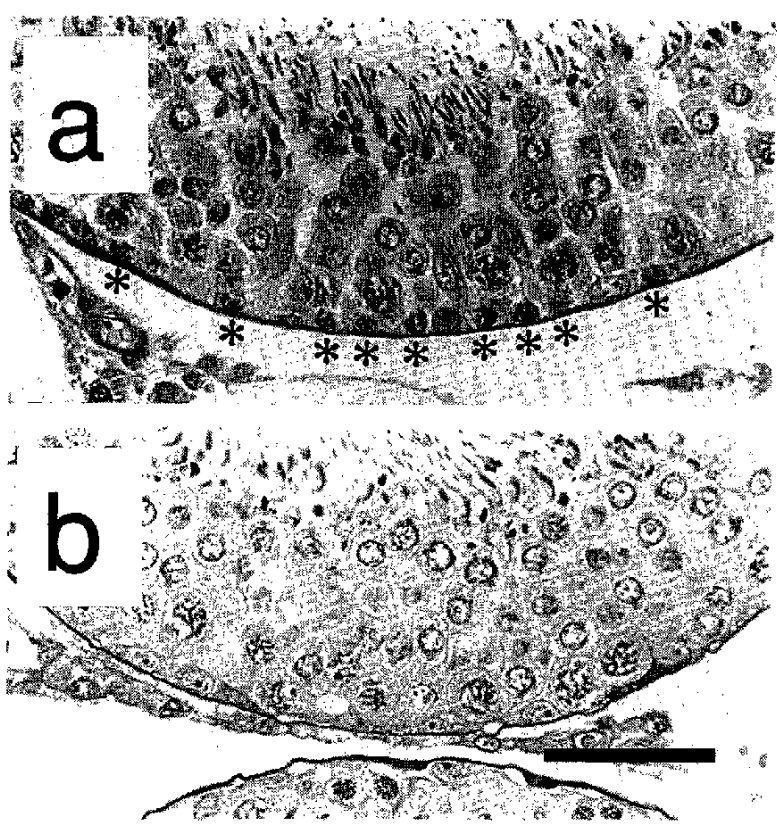

Fig. 2. Seminiferous epithelium at stage VII in control rat (a) and the rat treated with $1,355 \mathrm{mg} / \mathrm{kg}$ of 2 . bromopropane by s.c. injection five days a week for two weeks (b). In control rat, preleptotene spermatocytes (asterisk) are observed at the base of the epithelia (a). In the 2-bromopropane-treated rat, no preleptotene spermatocyte is observed (b). Bar indicates $50 \mu \mathrm{m}$.

Table 3. Numbers of germ cells in the seminiferous tubule in the control rats

\begin{tabular}{lcc}
\hline Type of germ cell & Stage & $\begin{array}{c}\text { Number } \\
\text { (/Sertoli cell } \\
\text { nucleus } / \text { tubule })\end{array}$ \\
\hline Spermatogonia & VII & $0.166 \pm 0.047$ \\
& X & $0.283 \pm 0.067$ \\
& XII & $0.223 \pm 0.014$ \\
I & $0.407 \pm 0.019$ \\
Spermatocyte & V & $1.452 \pm 0.070$ \\
Preleptotene & VII & $2.546 \pm 0.256$ \\
Leptotene & X & $2.509-0.401$ \\
Zygotene & XII & $2.573 \pm 0.193$ \\
Pachytene & I & $2.751 \pm 0.162$ \\
Pachytene & V & $3.329 \pm 0.463$ \\
Pachytene & VII & $3.474 \pm 0.431$ \\
Pachytene & X & $3.398 \pm 0.379$ \\
Pachytene & XII & $3.375 \pm 0.313$ \\
Round spermatid & & \\
& I & $8.336 \pm 0.405$ \\
& V & $9.346 \pm 0.976$ \\
& VII & $10.226 \pm 1.220$ \\
\hline
\end{tabular}

Results are expressed as meun $\pm \mathrm{SD}(\mathrm{n}=4)$.

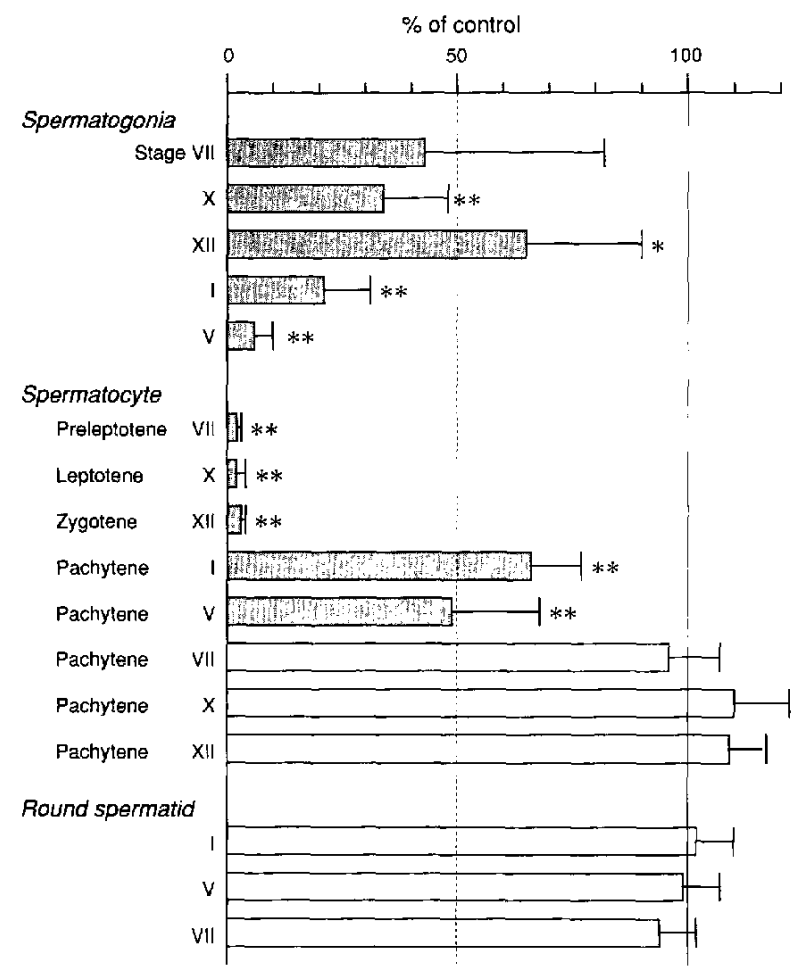

Fig. 3. Numbers of germ cells in the serniniferous tubule in the rats treated with $1,355 \mathrm{mg} / \mathrm{kg}$ of 2-bromopropane by s.c. injection five days a week for two weeks. Data are arranged according to the process of spermatogenesis and are expressed as \% of mean control value $(n=4)$. Bar indicates SD. Statistical significance is analyzed with Student's t-test; ${ }^{*} \mathrm{p}<0.05,{ }^{*} \mathrm{p}<0.01$

Fig. 3 shows the numbers of these germ cells in the 2-BP group. In Table 3, the number of germ cell is expressed as a number per one Sertoli cell nucleus per tubule. In Fig. 3, the number of germ cell is expressed as percentage of mean control value and is arranged according to the process of spermatogenesis. In the 2-BP group (Fig. 3), 40\%$60 \%$ decrease was observed in the number of spermatogonia at stages VII, $\mathrm{X}$ and XII (type $\mathrm{A}$ spermatogonia). The decrease in the number of spermatogonia became more prominent at stage I (type $\mathrm{A}$ and intermediate type spermatogonia, $80 \%$ decrease) and stage $\mathrm{V}$ (type $\mathrm{A}$ and type $\mathrm{B}$ spermatogonia, $95 \%$ decrease), and $96 \%-98 \%$ decrease was observed in the numbers of preleptotene spermatocyte, leptotene spermatocyte and zygotene spermatocyte at stages VII, X and XII, respectively. Significant decrease was still observed in the number of pachytene spermatocyte at stages $I$ and $V$. But the numbers of pachytene spermatocyte at the latter stages and round spermatids were comparable with those in control group.

In some seminiferous tubules at stages I-IV, there was a mass degeneration of pachytene spermatocyte 


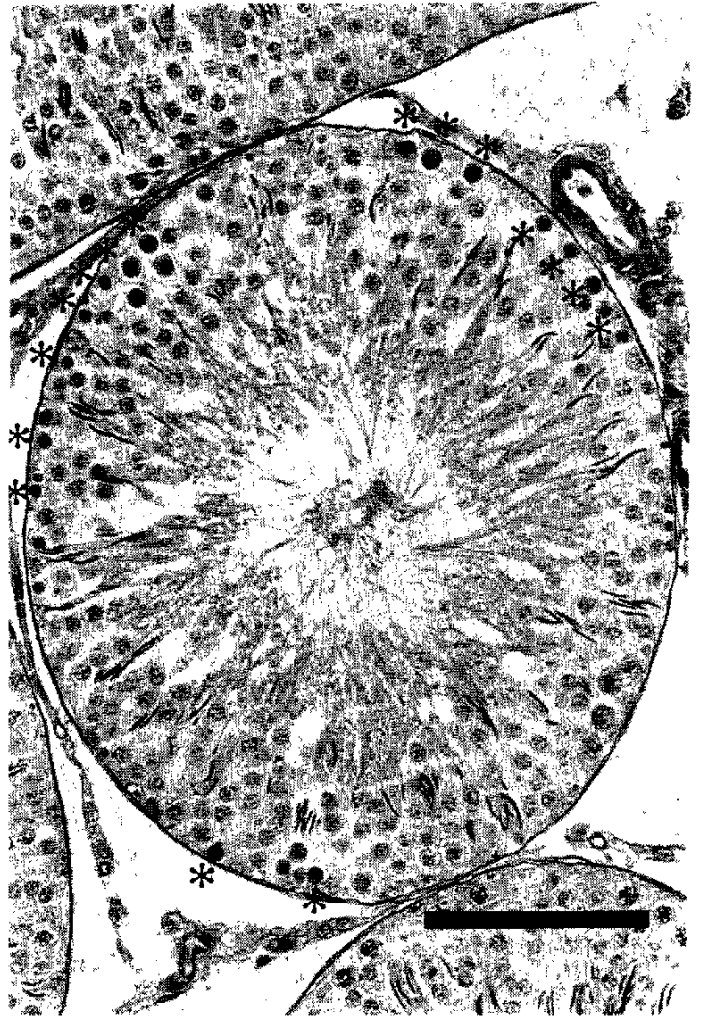

Fig. 4. Seminiferous tubule at stage IV in the rat treated with $1,355 \mathrm{mg} / \mathrm{kg}$ of 2-bromopropane by $\mathrm{s.c}$ injection five days a week for two weeks. There is a mass degeneration of pachytene spermatocyte (asterisk). Bar indicates $100, \mu \mathrm{m}$.

in the 2-BP group. Figure 4 shows the seminiferous tubule at stage IV in the 2-BP-treated rat. Many pachytene spermatocyte (asterisk) shrunk slightly and were densely stained. These morphological changes indicates germ cell degeneration. But even in the 2-BP-treated rats, there were many tubules which contained no degenerating pachytene spermatocytes at these stages.

\section{Discussion}

In our rapid communication ${ }^{8)}$, we reported 2-BPinduced decrease in the numbers of spermatogonia and four types of spermatocytes in the testis stained with hematoxylin and eosin. In the 2-BP-treated rats, the number of spermatogonia decreased at all stages examined (stages $\mathrm{X}, \mathrm{XII}$ and $\mathrm{I}$ ) and the numbers of preleptotene spermatocyte (stage VII) leptotene spermatocyte (stage $\mathrm{X}$ ) and zygotene spermatocyte (stage XII) decreased, too. As for pachytene spermatocyte, the number decreased at stage I but did not decrease at stage VII in these rats. But we could not evaluate a change in the number of pachytene spermatocyte at stages II $\cdot$ III$\mathrm{VI}$ in the previous examination because acrosome of the spermatid head, which is an important feature to determine the stage of the seminiferous tubule, is unstained in hematoxylin-eosin-stained section ${ }^{13}$, and individual stage was indistinguishable at stages II III-VI. This time, we stained the testicular section with periodic acid Schiff reagent (PAS), which is commonly used to stain acrosome, and evaluated a change in the numbers of spermatogonia, four types of spermatocytes and round spermatids at stages I, VII, X, XII and V. Similar to pachytene spermatocyte at stage $I$, the number of pachytene spermatocyte decreased at stage $\mathrm{V}$ in the 2-BP treated rats. In addition, like pachytene spermatocyte at stage VII, the numbers of pachytene spermatocyte at stages $X$ and $X I I$ and round spermatids did not decrease in these rats. In summary, the numbers of spermatogonia, preleptotene spermatocyte, leptotene spermatocyte and pachytene spermatocyte at stages $\mathrm{I}-\mathrm{V}$ decreased after the subcutaneous injection of 2-BP for two weeks. Howev$\mathrm{er}$, this result does not mean that all of these germ cells were initially affected by 2-BP. Germ cells in the seminiferous tubules are developing continuously in the process known as spermatogenesis. Figure 5 shows the development of spermatogonia at stages I-VI. This figure is made based on the duration of one cycle of the seminiferous epithelium (12.9 days) and the length of individual stages in rats by Clermont and Harvey ${ }^{10}$. For example, intermediate type spermatogonia at stage II ' III develop to preleptotene or leptotene spermatocytes at stages VIII$X I$ after 7 days and develop to pachytene spermatocytes at stages II-III $\mathrm{V}$ after 14 days. Therefore, if one type of germ cell suffers from an adverse effect and degenerates, a decrease in the number of germ cell appears not in this germ cell but in more developed germ cell after several days. According to Fig. 5, spermatocytes develop to pachytene spermatocyte at stage VII or more developed germ cells after 14 days. In this study, the number of pachytene spermatocyte decreased at stage $\mathrm{V}$ but did not decrease at stage VII after two-

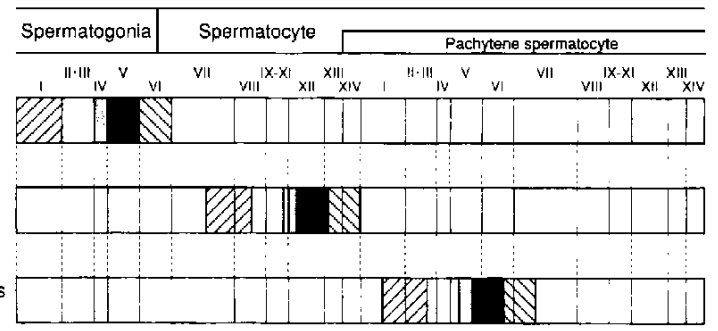

Fig. 5. Spermatogenic development of spermatogonia at stage I

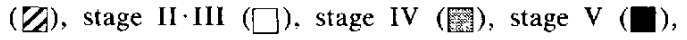
and stage VI $(\mathbb{N})$, Preleptotene spermatocyte appears in the latter period of stage VI. 
week treatment of 2-BP. Assuming that 2-BP affected germ cells immediately after the injection, it seemed likely that 2-BP had no adverse effects on spermatocytes but affected spermatogonia. In another study, we found a decrease in the number of spermatogonia in rats within $24 \mathrm{hr}$ after a subcutaneous injection of 2-BP (unpublished data). Therefore, we estimated that spermatogonia were the target cells of 2-BP in the testis in rats. There are roughly three types of spermatogonia in the seminiferous epithelium in rats, type A spermatogonia, intermediate type spermatogonia and type B spermatogonia. There are only type $\mathrm{A}$ spermatogonia at stages VII, $\mathrm{X}$ and XII, type $\mathrm{A}+$ intermediate type spermatogonia at stage $I$ and type $A+$ type $B$ spermatogonia at stage $\mathrm{V}^{12}$. Compared with the control value, the number of spermatogonia decreased at stages VII, X and XII, but the degrees were not severer than those at stages $I$ and $V$ in the 2-BP treated rats (Fig. 3). We did not distinguish these three types of spermatogonia in this study. But this result probably indicated that type A spermatogonia were less vulnerable to 2-BP treatment than intermediate type and type $B$ spermatogonia.

In some seminiferous tubules at stages I-IV, there was a mass degeneration of pachytene spermatocyte in the 2 -BP-treated rats. If these pachytene spermatocytes were initially affected by 2 -BP treatment, the number of round spermatids decreased at the sacrifice (see Fig. 5). But actually, the number of round spermatids did not decrease in the 2-BPtreated rats (Fig. 3). Therefore, we did not estimate that pachytene spermatocytes at stages I-IV were primary target cells of 2-BP. Probably, this histopathological change appeared immediately before the sacrifice because Sertoli cells promptly phagocytize degenerated germ cells and eliminate them from the seminiferous epithelium.

Ichihara et al. reported a decrease in the epididymal sperm count and severe atrophy of the seminiferous tubule after nine-week exposure to $1,000 \mathrm{ppm}$ of $2-\mathrm{BP}^{4}$. Epididymal sperm count did not decrease and there were few seminiferous tubules which became atrophic by two-week subcutaneous injection of $1,355 \mathrm{mg} / \mathrm{kg}$ of $2-\mathrm{BP}$ in this study. We sacrificed the rats at the next day of the last injection. However, if we had sacrificed the 2 -BP treated rats a few weeks after the last injection, the numbers of more developed germ cells would have decreased and the seminiferous tubules would have become atrophic because we found a severe decrease in the numbers of spermatogonia and spermatocytes in the 2-BP treated rats in this study. Therefore, the results of this study were not incon- sistent with those of the study of Ichihara et al.

In conclusion, from histopathological changes of the testis after two-week subcutaneous injection of 2-BP, we estimated that spermatogonia were the target cells of 2-BP in the testis in rats. Further studies are needed to confirm adverse effects of 2 $\mathrm{BP}$ on spermatogenesis in detail.

Acknowledgments: The authors thank Ms. Yoshiko Hirose for her technical assistance.

\section{References}

1) Kim Y, Jung K, Hwang T, et al. Hematopoietic and reproductive hazards of Korean electronic workers exposed to solvents containing 2 -bromopropane. Scand J Work Environ Health 1996; 22 : 387-391.

2) Park JS, Kim Y, Park DW, et al. An outbreak of hematopoietic and reproductive disorders due to solvents containing 2-bromopropane in an electronic factory, South Korea: Epidemiological survey. J Occup Health 1997; 39 : 138-143.

3) Ichihara G, Asaeda N, Kumazawa $T$, et al. Testicular toxicity of 2 -bromopropane. J Occup Health 1996; 38: 205-206.

4) Ichihara G, Asaeda $N$, Kumazawa $T$, et al. Testicular and hematopoietic toxicity of 2-bromopropane, a substitute for ozone layer-depleting chlorofluorocarbons. J Occup Health 1997; 39: 57-63.

5) Kamijima M, Ichihara G, Yu X, et al. Disruption in ovarian cyclicity due to 2-bromopropane in the rat. $\mathrm{J}$ Occup Health 1997; 39: 3-4.

6) Kim HY, Chung YH, Yi KH et al. $\mathrm{LC}_{50}$ of 2bromopropane. Ind Health 1996; 34: 403-407.

7) Maeng SH, Yu IJ. Mutagenicity of 2-bromopropane. Ind Health 1997; 35: 87-95.

8) Omura $M$, Zhao $M$, Romero $Y$, et al. Toxicity of 2bromopropane on spermatogonia and spermatocyte. $J$ Occup Health 1997; 39: 1-2.

9) Manderly JL, Tesarek JE, Sifford LJ, et al. Respiratory measurements of unsedated small laboratory mammals using nonbreathing valves. Lab Anim Sci $1979 ; 29: 323-329$.

10) Clermont $Y$, Harvey SC. Duration of the cycle of the seminiferous epithelium of normal, hypophysectomized and hypophysectomized-hormone treated albino rats. Endocrinol 1965; 76: 80-89.

11) Mori K, Kaido M, Fujishiro K, et al. Dose dependent effects of inhaled ethylene oxide on spermatogenesis in rats. Brit $J$ Ind Med 1991; 48: 270274.

12) Russell LD, Ettlin RA, Sinha-Hikim AP, Clegg ED. Histological and histopathological evaluation of the testis. Clearwater: Cache River Press, 1990: 62-118.

13) Clermont Y, Perey B. The stages of the cycle of the seminiferous epithelium of the rat: Practical definitions in PA-Schiff-hematoxylin and hematoxylineosin stained sections. Rev Canad Biol 1957; 16 : 451462. 